\title{
Probability distribution of the conductance at the mobility edge
}

\author{
Peter Markoš \\ Institute of Physics, Slovak Acad. Sci, Dúbravská cesta 9, 84228 Bratislava, Slovakia
}

June 18, 2018

\section{Abstract}

Distribution of the conductance $P(g)$ at the critical point of the metal-insulator transition is presented for three and four dimensional orthogonal systems. The form of the distribution is discussed. Dimension dependence of $P(g)$ is proven. The limiting cases $g \rightarrow \infty$ and $g \rightarrow 0$ are discussed in detail and relation $P(g) \rightarrow 0$ in the limit $g \rightarrow 0$ is proven

PACS numbers: 71.30.+h, 71.23.-k, 72.15.Rn

As the conductance $g$ in disordered systems is not the self-averaged quantity, the knowledge of its probability distribution is extremely important for our understanding of transport. This problem is of special importance at the critical point of the metal-insulator transition [1]. While the distribution of the conductance in the metallic phase is known to be Gaussian in agreement with the random-matrix theory [2] and the localized regime is characterized by the log-normal distribution of $g$ [2], the form of the critical distribution remains still unknown. Among the problems which are not solved yet we mention e.g. the number of parameters which characterize distribution, the existence of huge fluctuations of the conductance, and the form of $P(g)$ for small values of $g$.

Several attempts has been made to characterize conductance distribution at the critical point. Using the Migdal - Kadanoff renormalization treatment, huge conductance fluctuations has been predicted in [3]. The same conclusion was found also in systems of dimension $d=2+\varepsilon$. In the limit $\varepsilon<<1$ the form of the

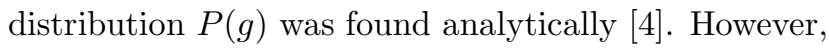
numerical studies of disordered 3D system 5 indicated that it is not possible to generalize these analytical conclusions for realistic $3 \mathrm{D}$ systems $(\varepsilon=1)$.

The form of $P(g)$ for $2 \mathrm{D}$ symplectic models was found in [6, 7]. Recently, $P(g)$ has been studied also for system in magnetic field, both in $3 \mathrm{D}$ [8] and in $2 \mathrm{D}$
19]. The main conclusion of these studies is that the symmetry of the system influences the form of the distribution at the critical point more strongly than in the metallic or localized regime. Nevertheless, $P(g)$ is invariant with respect to the choice of the microscopic model within the same universality class [7].

Studies of the statistics of the conductance have their counterpart in the analysis of the level statistics $s=E_{i+1}-E_{i}$ of the eigenvalues of Hamiltonian [11]. The critical distribution $P(s)$ is also the subject of intensive studies within last years 12 . In particular, its dependence on the symmetry [13], and dimension 14] have been studied numerically.

In this Letter we present new numerical data for the 3D and 4D Anderson model (orthogonal ensembles). Although data prove the dimension dependence of the distribution, their enables us to discuss the common features of the critical distribution. In particular, we prove that $P(g)$ decreases more quickly than exponentially for large $g$. This assures that there are no huge fluctuations of the conductance, discussed in [3]. We prove also that $P(g) \rightarrow 0$ in the limit of $g \rightarrow 0$.

We calculated the conductance as

$$
g=\operatorname{Tr} t^{\dagger} t=\sum \cosh ^{-2}\left(z_{i} / 2\right)
$$

where quantities $z_{i}$ determine eigenvalues of the transmission matrix $t^{\dagger} t$. Details of the method have been published elsewhere [5]. For a given system size $L$, the probability distribution of $g$ has been calculated from an ensemble of $N_{\text {stat }}$ samples. The list of used ensembles together with mean and variances of $g$ are given in Table 1.

The last column of Table 1 presents parameter $\left\langle z_{1}\right\rangle$, which corresponds to the parameter $\Lambda$ introduced in the finite size scaling theory by MacKinnon and Kramer 15] as $\left\langle z_{1}\right\rangle=\frac{2 L_{t}}{L \Lambda}$ in the quasi-one dimensional limit $L^{d-1} \times L_{t}, L_{t}>>L$. When neglecting the smallest system size, our data confirm the $L$-invariance 


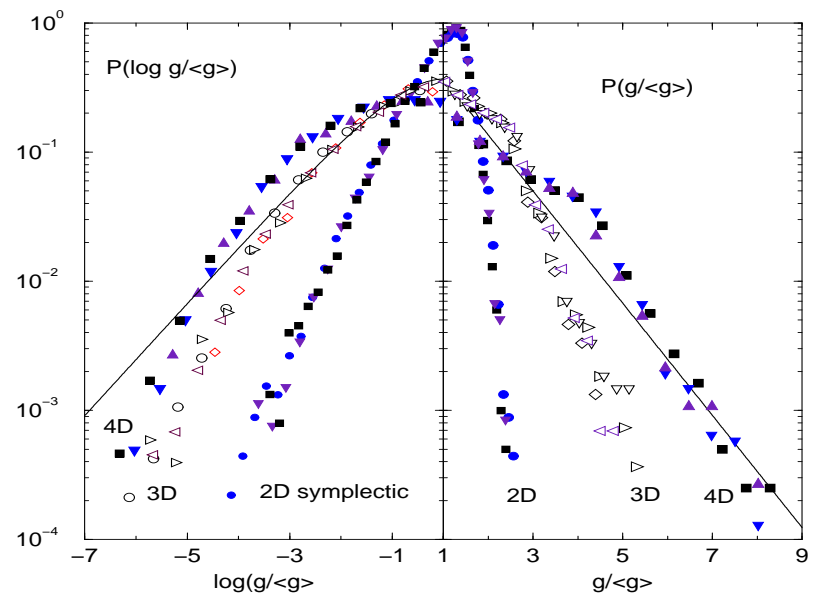

Figure 1: Probability distribution of $\log g /\langle g\rangle$ (left) and $g /\langle g\rangle$ (right) for 4D (full symbols) and 3D (open symbols) Anderson model. For comparison, we plot also data for 2D (symplectic) Ando model. The last exhibit the best convergence for both small and large values of $g$. For meaning of symbols, see Table 1 . Solid line is Poisson distribution $P(g)=\exp -g /\langle g\rangle$.

of $\left\langle z_{1}\right\rangle$ as well as of $\langle g\rangle$ and $\langle\log g\rangle$ and their standard deviations. Owing to higher critical disorder, $\left\langle z_{1}\right\rangle$ is larger in $4 \mathrm{D}$ than in $3 \mathrm{D}$. This guarantees that the finitesize effects disappear more quickly in $4 \mathrm{D}$. Therefore, in spite of the fact that computer facilities limited the system size to $L \leq 8$ for $d=4$, obtained data provide us with the relevant information about all parameters of interest.

We presented in Table 1 both mean values of $g$ and $\log g$ to underline the common features of $3 \mathrm{D}$ and $4 \mathrm{D}$ distribution: the variance of $\log g$ is of order of its mean value. This relation is typical for localized state. On the other hand, standard deviation of $g$ is also $\sim\langle g\rangle$. Its value for $3 \mathrm{D}$ samples, 0.334 , is smaller than the same quantity calculated for $3 \mathrm{D}$ in the metallic regime [5].

Numerical data for $P(g)$ are presented in Figure 1. They confirm that the critical distribution of $g$ is system-size independent, in agreement with previous studies. Fig. 1. shows also that $P(g)$ depends on the dimension of the system within the same symmetry class. Although the distribution has the same form for $3 \mathrm{D}$ and $4 \mathrm{D}$ ensembles, it becomes broader for higher $d$ : the probability to find $g<\langle\langle g\rangle$ or $g\rangle>\langle g\rangle$ growths with dimension. This is due to higher critical disorder, which causes that electronic state posses more features
Table 1: Review of ensembles studied in the present work. $L$-size of the $d$-dimensional cube, $N_{\text {stat }}$ : number of samples in a given ensemble, $\operatorname{var} g=\langle g\rangle^{2}-\left\langle g^{2}\right\rangle,\left\langle z_{1}\right\rangle$ is mean of the smallest of $z$ 's. Data for 3D AM are in good agreement with [9] (up to the spin degeneracy factor 2).

\begin{tabular}{|c|c|c|c|c|c|c|c|}
\hline $\bar{L}$ & & $N_{\text {stat }}$ & $\langle g\rangle$ & $\sqrt{\operatorname{varg}}$ & $\langle\log g\rangle$ & var $\log g$ & $\left\langle z_{1}\right\rangle$ \\
\hline \multicolumn{8}{|c|}{ 3D Anderson model: $W_{c} \approx 16.5$} \\
\hline 6 & $\circ$ & 20.000 & 0.375 & 0.324 & -1.481 & 1.344 & 2.901 \\
\hline 8 & $\triangleleft$ & 20.000 & 0.400 & 0.333 & -1.384 & 1.251 & 2.803 \\
\hline 10 & $\triangleright$ & 10.000 & 0.410 & 0.337 & -1.347 & 1.229 & 2.770 \\
\hline 12 & $\diamond$ & 5.000 & 0.421 & 0.340 & -1.302 & 1.199 & 2.724 \\
\hline 14 & $\triangle$ & 2.500 & 0.416 & 0.338 & -1.306 & 1.122 & 2.725 \\
\hline 18 & $\nabla$ & 500 & 0.418 & 0.329 & -1.279 & 1.083 & 2.717 \\
\hline \multicolumn{8}{|c|}{ 4D Anderson model: $W_{c} \approx 34.5[12]$} \\
\hline 4 & & 22.000 & 0.190 & 0.247 & -2.569 & 2.301 & 4.130 \\
\hline 5 & $\nabla$ & 30.000 & 0.229 & 0.270 & -2.275 & 2.006 & 3.838 \\
\hline 6 & $\square$ & 15.000 & 0.225 & 0.269 & -2.291 & 2.054 & 3.852 \\
\hline 7 & $\triangle$ & 7.000 & 0.239 & 0.275 & -2.193 & 1.971 & 3.748 \\
\hline 8 & & 200 & 0.227 & 0.274 & -2.188 & 1.692 & 3.790 \\
\hline
\end{tabular}

of the localized state than that of the metallic one (remaining critical). This is in agreement with studies of the level statistics in 4D [12].

The small- $g$ behavior of $P(g)$ can be estimated from Fig 1. Instead of $P(g /\langle g\rangle)$, we plot in the left side of Fig. 1. the distribution $\mathcal{P}(\gamma)$ of $\gamma=\log g /\langle g\rangle$. Evidently, $\log \mathcal{P}(\gamma)=\gamma+\log P(\exp \gamma)$. Therefore, an assumption $P(g=0)=c \neq 0$, implies $\mathcal{P}=\gamma+\log c$ for $\gamma \rightarrow-\infty$.

Fig 1 . shows clearly that $\log \mathcal{P}(\gamma)$ decreases more quickly than $\gamma$ for all ensembles we consider. This guarantees that $P(g) \rightarrow 0$ as $g \rightarrow 0$. Let us note that it is almost impossible to obtain last result form the studies of $P(g)$ on the linear scale 10 .

The small- $g$ behavior of $P(g)$ is easy to estimate also from the distribution $P\left(z_{1}\right)$ of the smallest parameter $z_{1}$. Indeed, small values of $g$ require large values of $z_{1}$. Neglecting contributions of other channels, we have

$$
\frac{1}{2 \varepsilon} \int_{0}^{2 \varepsilon} P(g) d g=\frac{1}{2 \varepsilon} \int_{\tilde{z}_{1}}^{\infty} P\left(z_{1}\right) d z_{1}
$$

with $\varepsilon=\exp -\tilde{z_{1}}$. In the limit $\varepsilon \rightarrow 0$ the integral on the LHS reads $\sim P(g), g=\varepsilon$. RHS could be found analytically for special form of $P\left(z_{1}\right)$. In particular, for Wigner surmises $P\left(z_{1}\right)=\pi / 2\left\langle z_{1}\right\rangle^{2} \times z_{1} \exp (-\pi / 4 \times$ $\left.\left[z_{1} /\left\langle z_{1}\right\rangle\right]^{2}\right)$ we obtain that $P(g) \sim g^{-1-\text { const } \times \log g} / 2$ with const $=\frac{\pi}{4\left\langle z_{1}\right\rangle^{2}}$. Consequently, $P(g=0)=0$. Fig. 2. assures that $P\left(z_{1}\right)$ decreases more quickly than 


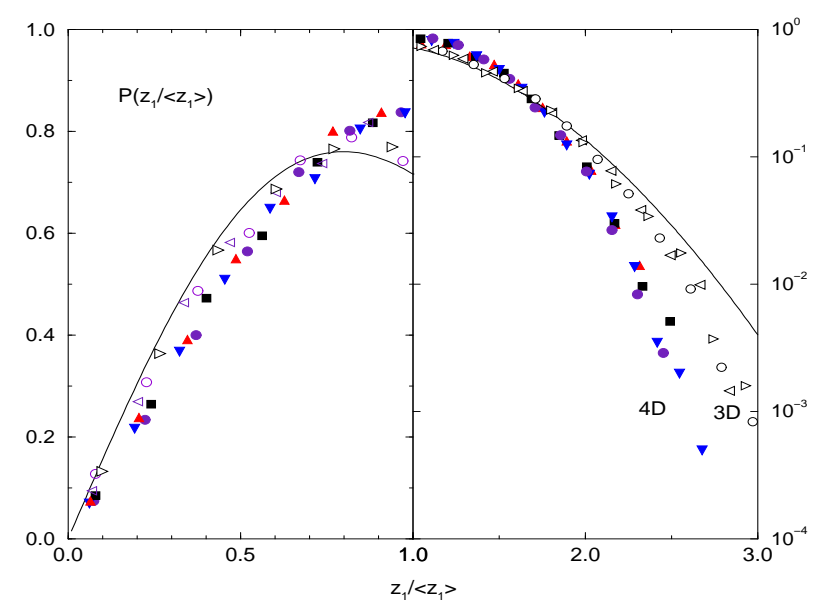

Figure 2: Probability distribution of (normalized) $z_{1}$ for 4D and 3D Anderson model. Solid line is Wigner surmises $P_{W}(z)=\frac{\pi}{2} z \exp \left[-\frac{\pi}{4} z^{2}\right]$. For the mean value $\left\langle z_{1}\right\rangle$ see Table 1 .

Wigner surmise for large $z_{1}$ in orthogonal ensemble for both $3 \mathrm{D}$ and $4 \mathrm{D}$ systems. This assures that $P(g) \rightarrow 0$ as $g \rightarrow 0$.

Linear behavior of the distribution $P\left(z_{1}\right)$ for small $z_{1}$ (see left side of Fig. 2) guarantees nonzero probability that the first channel is fully open. Indeed, if $P\left(z_{1}\right) \sim C \times z_{1}$ for $z_{1} \rightarrow 0$, then the probability, that the first channel contribution to the conductance, $g_{1}=1 / \cosh ^{2}\left(z_{1}\right)$, equals to 1 , is $C$. This explains the origin of the characteristic bump in the distribution $P(g)$ for $g=1$. In Fig. 1, the bump is clearly visible for both $3 \mathrm{D}$ and $4 \mathrm{D}$ systems.

Fig. 1 (right) confirms that $P(g)$ decreases more quickly than exponentially for large $g$. This is easy to understand on the basis of the analysis of the statistics of $z$ 's presented in [5]. Fig 3. shows mean values and variances of some smallest $z$ 's for both $3 \mathrm{D}$ and $4 \mathrm{D}$ system. Evidently, $\left\langle z_{i}\right\rangle \sim \mathcal{O}(1)$ and variances var $z_{i}$ decreases quickly with index $i$. Consequently, the contribution to the conductance from the second (higher) channel is, due to (11), small (negligible). To estimate this contribution, we note that all higher $z_{i}, i \geq 2$, are normally distributed. [5]. Their mean and variances has been estimated as $\left\langle z_{i}\right\rangle \sim\left\langle z_{1}\right\rangle \times i^{1 /(d-1)}$ and var $z_{i} \sim$ $\left\langle z_{i}\right\rangle^{-(d-2)}$ [16]. Although this result holds only in the quasi-one dimensional limit, where the mutual correlations of $z$ 's are negligible, they serve as a good quantitative estimation also for true $d$-dimensional cubes. As $\mathrm{i}$ is seen in Figure 3, this agreement is better for 4D

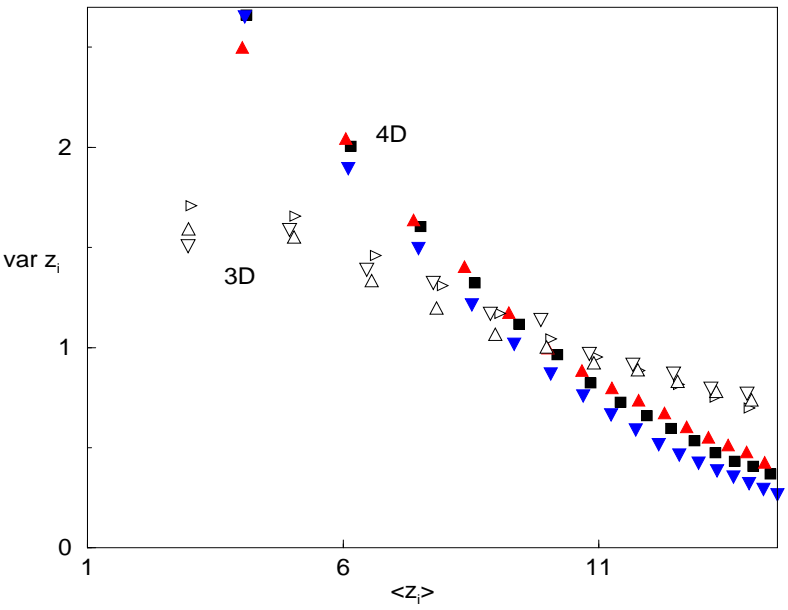

Figure 3: Var $z_{i}$ as a function of $\left\langle z_{i}\right\rangle$ (only for $\langle z\rangle<15$ ) for 3D (open symbols) and 4D (full symbols) orthogonal systems. Note the system-size invariance of presented parameters (at least for $i \leq L$ ).

than for $3 \mathrm{D}$,. Then, the probability to find $g \approx n$ is less than $\exp \left[-\left\langle z_{n}\right\rangle / 2 \operatorname{var} z_{n}\right] \sim \exp \left[-\right.$ const $\left.\times n^{d /(d-1)}\right]$ and

$$
P(g) \sim \exp -\text { const } \times g^{d /(d-1)} .
$$

We conclude that presented numerical data for $3 \mathrm{D}$ and 4D Anderson model prove the system size invariance of the conductance distribution at the critical point. Although the distribution depends on the dimension and symmetry of the system, we found its common features, namely exponential decrease of $P(g)$ for $g>1$, and a decrease of $P(g)$ to zero for $g=0$. We show that the form of $P(g)$ can be analyzed on the basis of the statistics of parameters $z$ introduced by relation (1). This analysis is more simple for higher dimension, where the statistical correlations of $z \mathrm{~s}$ are supposed to be less important.

Acknowledgement This work has been supported by Slovak Grant Agency, Grant n. 2/4109/98.

\section{References}

[1] B. Shapiro, Phys. Rev. Lett. 651510 (1990)

[2] J.-L. Pichard, Quantum Coherence in Mesoscopic Systems, edited by B. Kramer, NATO ASI Ser. B 254 (New York: Plenum) p. 369 (1991)

[3] A. Cohen, Y. Roth and B. Shapiro, Phys. Rev. B 3812125 (1988) 
[4] A. Cohen and B. Shapiro, Int. J. Mod. Phys. B 6, $1243(1992)$

[5] P. Markoš and B. Kramer, Phil. Mag. B. 68, 357 (1993).

[6] P. Markoš, J. Phys. I, 4, 551 (1994).

[7] P. Markoš, Europhys. Lett. 26, 431 (1994)

[8] K. Slevin and T. Ohtsuki, Phys. Rev. Lett. 78, 4083 (1987).

[9] Xiashoa Wang, Quiming Li, C.M. Soukoulis, condmat/9803356

[10] C.M. Soukoulis, Xiaosha Wang, Qiming Li and M.M. Sigalas, comment to PRL (condmat/987410).

[11] B. I. Shklovskii, B. Shapiro, B.R. Sears, P. Lambrianides, H.B. Shore, Phys. Rev. B 47, 11487 (1993)

[12] I.Kh. Zharekeshev and B. Kramer, Phys. Rev. Lett. 79, 717 (1997)

[13] M. Batsch, L. Schweitzer, I. Kh. Zharekeshev, B. Kramer, Phys. Rev. Lett. 77, 1552 (1996)

[14] I.Kh. Zharekeshev and B. Kramer, to appear in Ann.Phys. (cond-matt/9810286)

[15] A. MacKinnon and B. Kramer, Phys. Rev. Lett. 471546 (1981)

[16] P. Markoš, J. Phys.: Condens. Matt. 7, 8361 (1995)

[17] P. Markoš, J. Phys.A: Math. Gen. 303441 (1997) 